Acta Crystallographica Section D

\section{Biological Crystallography}

ISSN 0907-4449

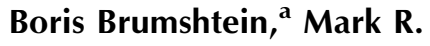
Wormald, ${ }^{\text {b }}$ Israel Silman, Anthony H. Futerman ${ }^{d}$ and Joel L. Sussman ${ }^{\mathrm{a} *}$

aDepartment of Structural Biology, Weizmann Institute of Science, Israel, ${ }^{\mathbf{b}}$ Oxford

Glycobiology Institute, Department of Biochemistry, University of Oxford, Oxford OX1 3QU, England, 'Department of Neurobiology, Weizmann Institute of Science, Israel, and ${ }^{\mathbf{d}}$ Department of Biological Chemistry, Weizmann Institute of Science, Israel

Correspondence e-mail:

joel.sussman@weizmann.ac.il

\title{
Structural comparison of differently glycosylated forms of acid- $\beta$-glucosidase, the defective enzyme in Gaucher disease
}

Gaucher disease is caused by mutations in the gene encoding acid- $\beta$-glucosidase. A recombinant form of this enzyme, Cerezyme ${ }^{\circledR}$, is used to treat Gaucher disease patients by 'enzyme-replacement therapy'. Crystals of Cerezyme ${ }^{\mathbb{R}}$ after its partial deglycosylation were obtained earlier and the structure was solved to $2.0 \AA$ resolution [Dvir et al. (2003), EMBO Rep. 4, 704-709]. The crystal structure of unmodified Cerezyme ${ }^{\mathbb{R}}$ is now reported, in which a substantial number of sugar residues bound to three asparagines via $\mathrm{N}$-glycosylation could be visualized. The structure of intact fully glycosylated Cerezyme $^{\mathbb{R}}$ is virtually identical to that of the partially deglycosylated enzyme. However, the three loops at the entrance to the active site, which were previously observed in alternative conformations, display additional variability in their structures. Comparison of the structure of acid- $\beta$-glucosidase with that of xylanase, a bacterial enzyme from a closely related protein family, demonstrates a close correspondence between the active-site residues of the two enzymes.

\section{Introduction}

Gaucher disease, the most common lysosomal storage disorder (Futerman \& van Meer, 2004), is caused by mutations in the gene encoding acid- $\beta$-glucosidase (glucocerebrosidase, GlcCerase; EC 3.2.1.45; Beutler \& Grabowski, 2001), resulting in intracellular accumulation of glucosylceramide (GlcCer). Fig. 1 shows the reaction catalyzed by GlcCerase. GlcCerase is a 497 amino-acid residue enzyme with a molecular weight of $\sim 62 \mathrm{kDa}$ (Horowitz et al., 1989; Grabowski et al., 1990). Mutations in GlcCerase diminish activity either by reducing catalytic activity or by reducing its lysosomal concentration. In the former case the mutations affect turnover number, substrate affinity and/or activator binding and in the latter they compromise folding in the endoplasmic reticulum, resulting in proteasomal degradation of the protein (Sawkar et al., 2005). GlcCerase is activated in lysosomes by saposin $\mathrm{C}$ (SapC; Bruhn, 2005; Vaccaro et al., 1997), although neither the mechanism of activation nor the precise role of SapC are well understood (Bruhn, 2005).

We previously determined the three-dimensional structure of Cerezyme ${ }^{\circledR}$ (Premkumar et al., 2005; Dvir et al., 2003), a recombinant form of GlcCerase that is used in enzymereplacement therapy (ERT) for Gaucher disease patients (Jmoudiak \& Futerman, 2005). The protein consists of three non-contiguous domains, with the catalytic site located in domain III (residues 76-381 and 416-430), a $(\beta / \alpha)_{8}$ (TIM) barrel. Although the function of the two non-catalytic domains is unknown, mutations that cause Gaucher disease are found in all three domains.
Received 4 July 2006 Accepted 19 September 2006

PDB Reference: acid$\beta$-glucosidase, 2j25, r2j25sf 
Table 1

Data-collection statistics for GCase.

Values in parentheses are for the highest resolution shell (2.97-2.9 $)$.

\begin{tabular}{ll}
\hline Radiation wavelength $(\AA)$ & 0.9759 \\
Temperature $(\mathrm{K})$ & 100 \\
Space group & $C 222_{1}$ \\
Unit-cell parameters $(\AA)$ & $a=108.6, b=280.8, c=91.0$ \\
Resolution range $(\AA)$ & $40-2.9$ \\
No. of observed reflections & $228034(16759)$ \\
No. of unique reflections & $30978(2227)$ \\
Completeness $(\%)$ & $98.7(98.5)$ \\
$\langle I\rangle / \sigma(I)$ & $13.75(5.3)$ \\
$R_{\mathrm{mrgd}-F}(\%)$ & $10.6(27.0)$ \\
\hline
\end{tabular}

There are five putative glycosylation sites in GlcCerase, four of which are believed to be occupied (Asn19, Asn59, Asn146 and Asn270; Grace et al., 1994). In order to target GlcCerase to macrophages, the main cell type affected in Gaucher disease (Jmoudiak \& Futerman, 2005), and to enhance internalization by mannose receptors on the surfaces of the macrophages (Brady, 2006), production of Cerezyme ${ }^{\circledR}$ involves the sequential deglycosylation of GlcCerase, using $\alpha$-neuraminidase, $\beta$-galactosidase and $\beta$ - $N$-acetylglucosaminidase, to expose terminal mannose residues, leaving the core glycan, an oligosaccharide which consists of five sugars, namely two $N$-acetylglucosamines and three mannoses.

In our previous structure determinations (Premkumar et al., 2005; Dvir et al., 2003), Cerezyme ${ }^{\circledR}$ was partially deglycosylated prior to crystallization using $N$-glycosidase $\mathrm{F}$, which removes carbohydrate chains by cleaving the amide bonds between Asn residues and $N$-acetylglucosamine (GlcNAc; Han \& Martinage, 1992), but does not necessarily remove all carbohydrate chains from native proteins; a similar protocol was used to obtain another recently reported Cerezyme ${ }^{\circledR}$ structure (PDB code 2f61; Liou et al., 2006).

In order to alleviate concerns that partial deglycosylation might alter its three-dimensional structure, we have now solved the structure of intact Cerezyme ${ }^{\circledR}$ (GCase) obtained without $\mathrm{N}$-glycosidase $\mathrm{F}$ treatment. The crystals display the same space group as the partially deglycosylated Cerezyme ${ }^{\circledR}$
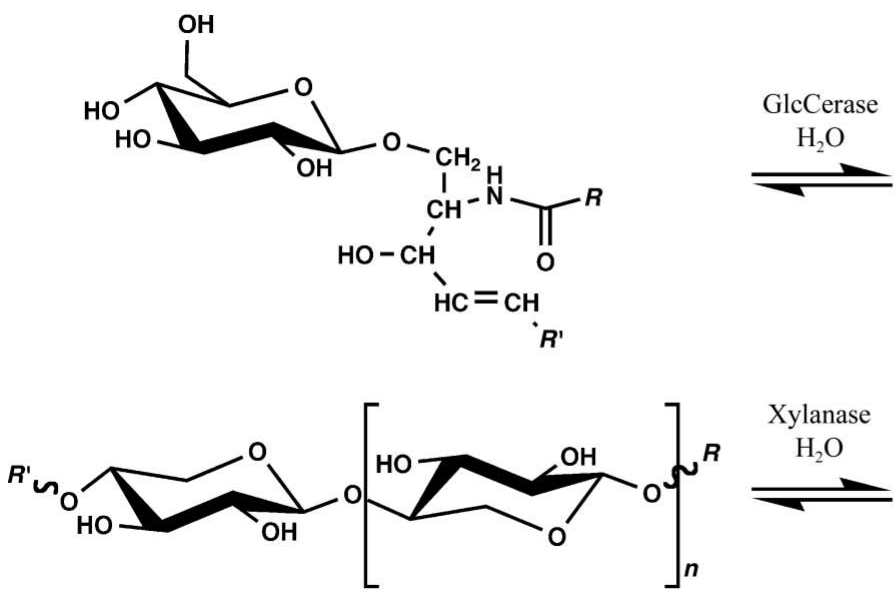

Figure 1

Reactions catalyzed by GlcCerase and xylanase.
(pDG-GCase); moreover, there are no fundamental differences between the two structures, although some novel conformations are observed in the lid region around the active site (Premkumar et al., 2005). In addition, we demonstrate that GlcCerase bears a strong structural similarity in its active-site region to xylanase (Larson et al., 2003), a glycoside hydrolase that shows the highest sequence similarity to GlcCerase among structures deposited in the PDB.

\section{Experimental procedures}

\subsection{Crystallization}

Cerezyme $^{\circledR}$ (GCase; Genzyme Corporation), obtained from patient leftovers, was dissolved in $100 \mathrm{~m} M \mathrm{NaCl}, 50 \mathrm{~m} M$ 2-(4-morpholino)ethanesulfonic acid (MES) $\mathrm{pH} 5.5$ at a concentration of $1-2 \mathrm{mg} \mathrm{ml}^{-1}$. The sample was washed with the same buffer and concentrated to $4-5 \mathrm{mg} \mathrm{ml}^{-1}$ in a Centricon device using a filter with a cutoff size of $30 \mathrm{kDa}$. GCase crystals were obtained by microbatch crystallization using a Douglas Instruments IMPAX I-5 robot. The crystallization solution contained a 1:1 ratio of the concentrated enzyme solution and $2 M\left(\mathrm{NH}_{4}\right)_{2} \mathrm{SO}_{4}, 0.1 M$ Bis-Tris pH 5.5. Crystallization was performed under oil (D'Arcy et al., 2003) for 5-14 d at 293 K. Data were collected on beamline ID23eh1 at the ESRF synchrotron facility in Grenoble, France. Crystals were mounted and flash-cooled at $100 \mathrm{~K}$. X-ray diffraction images were processed using XDS and XSCALE (Kabsch, 1993). Reflections were converted to a format suitable for REFMAC5 (Murshudov et al., 1997) using XDSCONV and processed in $\mathrm{CCP} 4$ (Collaborative Computational Project, Number 4, 1994). Table 1 summarizes data collection and processing.

\subsection{Structure determination and refinement}

Initial phases were obtained by molecular replacement using Phaser (McCoy et al., 2005) software. Molecule $A$ of pDG-GCase (PDB code 1ogs; Dvir et al., 2003) was used as a starting model for molecular replacement. REFMAC5 (Murshudov et al., 1997) was used for refinement and Coot
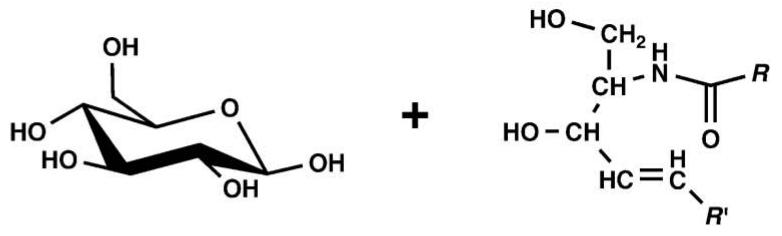

$\beta$-Glucose

Ceramide

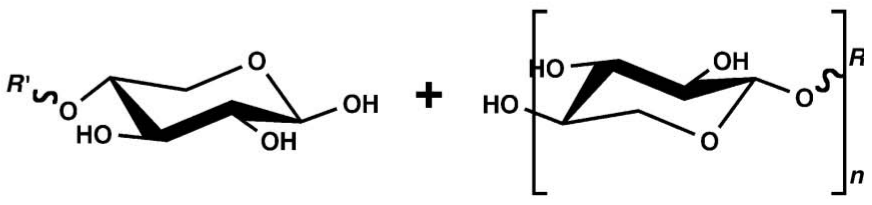


Table 2

Refinement statistics for the GCase structure.

\begin{tabular}{ll}
\hline Resolution range of refinement $(\AA)$ & $29.6-2.9$ \\
$R_{\text {work }} \dagger(\%)$ & 21.5 \\
$\mathrm{R}_{\text {free }}(\%)$ & 27.3 \\
Monomers per ASU & 2 \\
No. of different NCS groups & 2 \\
Average $B$ factor $\left(\AA^{2}\right)$ & 33.6 \\
R.m.s. deviations from ideal values & \\
$\quad$ Bond lengths $(\AA)$ & 0.015 \\
$\quad$ Bond angles $\left({ }^{\circ}\right)$ & 1.590 \\
$\quad$ Torsion angles $\left({ }^{\circ}\right)$ & 6.627 \\
Estimated coordinate error & \\
$\quad$ E.s.d. $\S$ from Luzzati plot & 0.38 \\
ESU based on $R$ free $(\AA)$ & 0.42 \\
ESU based on maximum likelihood $(\AA)$ & 0.31 \\
$\quad$ ESU for $B$ values based on maximum likelihood $\left(\AA^{2}\right)$ & 17.01 \\
Ramachandran outliers $(\%)$ & 0.2 \\
\hline
\end{tabular}

$\dagger R_{\text {work }}=\sum|| F_{\mathrm{o}}|-| F_{\mathrm{c}}|| / \sum\left|F_{\mathrm{o}}\right|$, where $F_{\mathrm{o}}$ denotes the observed structure-factor amplitude and $F_{\mathrm{c}}$ the structure-factor amplitude calculated from the model. $\$ R_{\text {free }}$ is for $5 \%$ of randomly chosen reflections excluded from the refinement. \& E.s.d., estimated standard deviation. - ESU, estimated standard uncertainty

(Emsley \& Cowtan, 2004) was used to build the model and fit it into the electron density. During refinement and model building, twofold noncrystallographic symmetry was applied, except for regions where different conformations were clearly detectable (Kleywegt, 1996). Residues 312-319 (loop 3) in molecule $B$ of the GCase model did not fit the electron density. Consequently, they were omitted from the refinement and rebuilt into a new conformation.

Electron-density maps revealed sugar residues attached to some of the Asn residues. Sugars were modelled into electron density according to the putative oligosaccharide sequences reported by the Genzyme Corporation for Cerezyme ${ }^{\circledR}$ (US patent Nos. 5236838 and 5549892; Murray, 1987). The conformations and angles of the oligosaccharides were confirmed with PDB-CARE (Lutteke \& von der Lieth, 2004) and CARP (Lutteke et al., 2005). Additional sugars, $\mathrm{SO}_{4}^{2-}$ ions and water molecules were added with improvement of electron-density maps and phases in subsequent refinement. Refinement results are summarized in Table 2.

\subsection{Comparison of the structures of GCase and xylanase}

A BLAST search for sequences in the PDB (Berman et al., 2000) was performed using the GCase sequence (Horowitz et al., 1989). The best fit was achieved for xylanase (EC 3.2.1.8; PDB code 1nof), a member of glycoside hydrolase family 5 (Larson et al., 2003). According to BLASTP (Altschul et al., 1997 ), the expectation value is $5 \times 10^{-3}$, with $18 \%$ identity and $41 \%$ similarity. The crystal structure of xylanase reveals a fold similar to that of GCase. Therefore, 1nof, which was determined to $1.42 \AA$ resolution (Larson et al., 2003), was used to cross-validate structural features of GCase (Keen et al., 1996).

\subsection{Validation and deposition}

The final models and structure factors of GCase were validated with PROCHECK (Laskowski et al., 1993) and deposited in the PDB with code $2 \mathrm{j} 25$.

\subsection{Modelling glycans on pDG-GCase}

Glycan modelling was performed on a Silicon Graphics Fuel workstation using INSIGHTII and DISCOVER software (Accelrys Inc., San Diego, USA). N-linked glycan structures were generated using the database of glycosidic linkage conformations (Wormald et al., 2002) and in vacuo energy minimization to relieve unfavourable steric interactions. The Asn-GlcNAc linkage conformations and analysis of the Asn side-chain conformations were based on the observed range of crystallographic values (Petrescu et al., 2004). The nomenclature for the Asn side-chain torsion angles is $\chi_{1}=\mathrm{N}-\mathrm{C}^{\alpha}-$ $\mathrm{C}^{\beta}-\mathrm{C}^{\gamma}, \chi_{2}=\mathrm{C}^{\alpha}-\mathrm{C}^{\beta}-\mathrm{C}^{\gamma}-\mathrm{N}^{\delta}$. Figures were produced with PyMol (http://www.pymol.org).

\section{Results}

\subsection{Crystallization}

Previous attempts to obtain Cerezyme ${ }^{\mathbb{R}}$ crystals without partial deglycosylation were unsuccessful (Dvir et al., 2003; Liou et al., 2006; Premkumar et al., 2005). In the current study, using a much larger screen of crystallization conditions (956 different conditions, excluding optimizations), employing the microbatch technique under oil (Chayen, 1998) and utilizing a Douglas Instruments IMPAX I-5 crystallization robot at room temperature, we were finally able to obtain crystals of Cerezyme $^{\circledR}$ without prior deglycosylation (GCase). To improve diffraction, the conditions which produced the best crystals were chosen for further optimization. GCase crystallizes in the same space group as previously reported for pDG-GCase, i.e. $C 222_{1}$, and with similar unit-cell parameters.

Analysis of the two structures indicates that all glycosylation sites are adjacent to empty cavities in the crystal, thus allowing placement of the sugars in these spaces without generating steric clashes that would hinder crystallization. The asymmetric unit of pDG-GCase was shown to contain two copies of the GlcCerase molecule, molecules $A$ and $B$, which, although very similar, are not completely identical to each other in conformation (Dvir et al., 2003; Premkumar et al., 2005). They also differ in the number of sugar molecules for which electron density can be assigned (Dvir et al., 2003). Some of the glycoside chains from adjacent asymmetric units make contacts with each other. These contacts stabilize and order those oligosaccharide chains in the crystal, thus making them visible in the electron-density maps.

The structure of GCase was refined to $2.9 \AA$ resolution. Some conformational differences were observed between molecules $A$ and $B$ in GCase and the corresponding molecules in pDG-GCase (PDB codes 1 ogs and 1y7v; Dvir et al., 2003; Premkumar et al., 2005). The changes seen are mainly in loops near the active site.

\subsection{Glycosylation of GCase}

Owing to crystal-packing constraints, the glycans in molecule $B$ are less mobile than those in molecule $A$ (Fig. $2 a$ ). Thus, in molecule $B$ a core glycan chain containing five sugar residues is seen attached to Asn19, three sugars are seen attached 
Table 3

Number of bound sugar residues observed in the crystal structures of GlcCerase.

\begin{tabular}{lllll}
\hline & Asn19 & Asn59 & Asn146 & Asn270 \\
\hline $2 \mathrm{j} 25-A$ & 2 & 0 & 1 & 0 \\
$2 \mathrm{j} 25-B$ & 5 & 3 & 2 & 0 \\
1 ogs- $A$ & 1 & 0 & 0 & 0 \\
1 ogs- $B$ & 2 & 0 & 0 & 0 \\
$1 \mathrm{y} 7 \mathrm{v}-A$ & 2 & 0 & 0 & 0 \\
$1 \mathrm{y} 7 \mathrm{v}-B$ & 2 & 0 & 0 & 0 \\
$2 \mathrm{f} 61-A$ & 1 & 0 & 0 & 0 \\
$2 \mathrm{f} 61-B$ & 2 & 0 & 0 & 0 \\
\hline
\end{tabular}

to Asn59, two to Asn146 and none to Asn270 (Table 3). In molecule $A$ only two sugars can be detected on Asn19, one on Asn146 and none on Asn59 and Asn270. The glycans attached

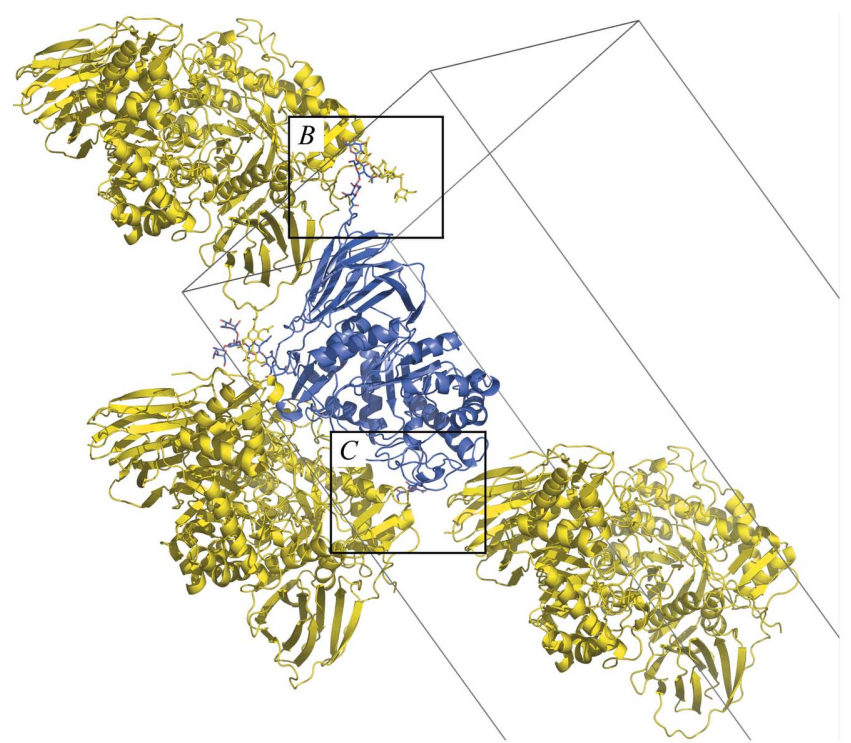

(a)

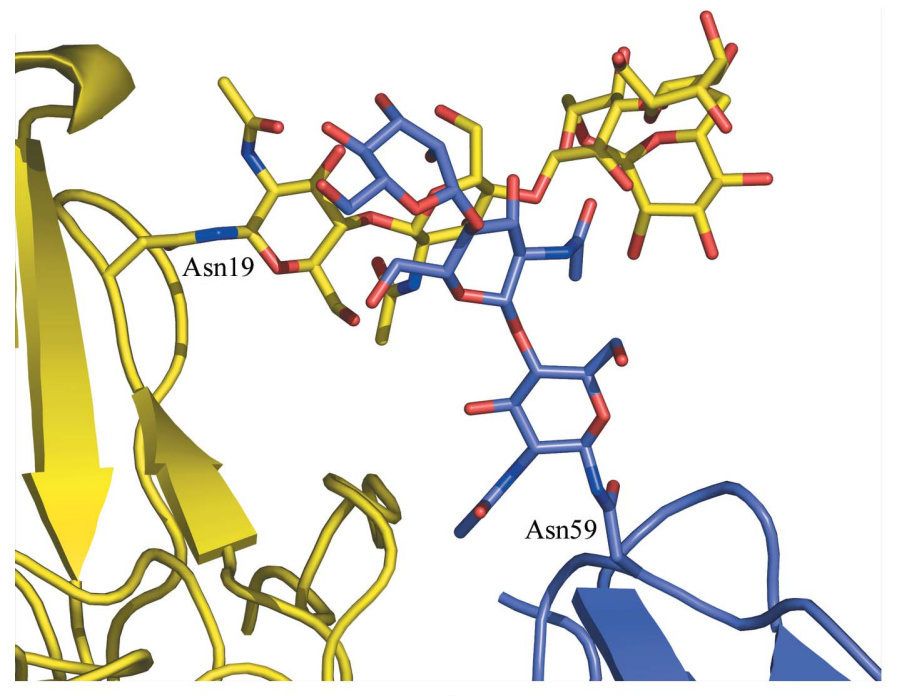

(b) to residue Asn19-B (i.e. Asn19 of molecule B) and to Asn59-B of a symmetry-related molecule are in contact with each other (Fig. 2b), causing the glycan chains to become ordered and, as a consequence, visible in the electron-density map. The two sugar moieties on the glycan of Asn146- $B$ do not make crystal contacts, consistent with the low number of sugar residues visible on Asn146 (Fig. 2c). The fact that we do not detect any sugars on residue Asn59- $A$ may be a consequence of the flexibility of this glycan in the crystal. Residues 59-64 adopt two different conformations in molecules $A$ and $B$ owing to different crystal contacts.

\subsection{Structural characteristics of GCase}

Some conformational differences, as distinguished by differences in $\varphi$ and $\psi$ angles (Kleywegt, 1996), were detected between molecules $A$ and $B$ of the asymmetric unit of GCase (Fig. 3).

3.3.1. Lid at the entrance to the active site. We previously described two loops in pDG-GCase whose conformations control access to the active site (Dvir et al., 2003; Premkumar et al., 2005). In molecule $A$ of GCase, we now detect conformational differences in an additional loop, loop 3 (residues 312-319; Table 4), that arise in conjunction with changes in the $\beta$-sheet structure of residues $341-344$, which are located adjacent to the catalytic residue Glu340 (Fig. 4). The rearrangement of loop 3 consists of side-chain flips of both Trp312 and Trp378 (Fig. 5), which are associated with the transition of the conformations of residues 312-319 and 341344 from a loop and a $\beta$-strand, respectively, to coils. However, these differences do not affect the conformation of the catalytic residues, since the distances between the side chains of Glu235 and Glu340 remain similar (Table 5). Moreover, the

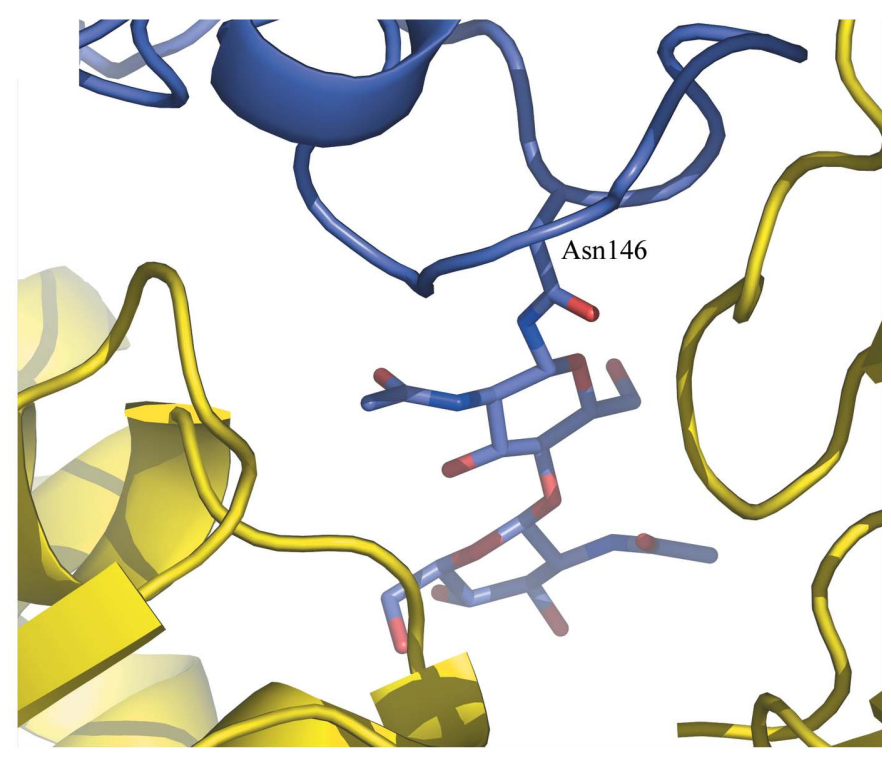

$(c)$

Figure 2

Packing of glycans in the GCase crystal. (a) Glycans in the cavities between the symmetry-related molecules in the crystal. Molecule $B$ of the asymmetric unit is shown in blue and the symmetry-related molecules are shown in yellow. The regions labelled $B$ and $C$ are magnified in $(b)$ and $(c)$, respectively. $(b)$ Magnification of a view of the sugars bound to Asn59 (blue) and to Asn19 on a symmetry-related molecule (yellow). (c) Magnification of a view of the sugars bound to Asn146 (blue). 
Table 4

Conformational classification of loops L1, L2 and L3 in the various GlcCerase crystal structures.

\begin{tabular}{|c|c|c|c|}
\hline Loop & L3, 312-319 & L1, 341-347 & L2, 393-399 \\
\hline \multicolumn{4}{|c|}{ Conformation } \\
\hline \multirow[t]{4}{*}{ Open $\dagger$} & $2 \mathrm{j} 25-B$ & $2 \mathrm{j} 25-A, 2 \mathrm{j} 25-B$ & $2 \mathrm{j} 25-B$ \\
\hline & 1ogs- $A, 1$ logs- $B$ & 1ogs- $A$ & logs $-B$ \\
\hline & $1 \mathrm{y} 7 \mathrm{v}-A, 1 \mathrm{y} 7 \mathrm{v}-B$ & & $1 \mathrm{y} 7 \mathrm{v}-A, 1 \mathrm{y} 7 \mathrm{v}-B$ \\
\hline & $2 \mathrm{f} 61-A, 2 \mathrm{f} 61-B$ & $2 \mathrm{f} 61-A$ & $2 \mathrm{f} 61-B$ \\
\hline \multirow[t]{4}{*}{ Closed $\dagger$} & $2 \mathrm{j} 25-A$ & & $2 \mathrm{j} 25-A$ \\
\hline & & 1ogs- $B$ & logs $-A$ \\
\hline & & $1 \mathrm{y} 7 \mathrm{v}-A, 1 \mathrm{y} 7 \mathrm{v}-B$ & \\
\hline & & $2 \mathrm{f} 61-B$ & $2 \mathrm{f} 61-A$ \\
\hline
\end{tabular}

$\dagger$ The assignment of loop conformations as open or closed is based on the criteria established for 1y7v (Premkumar et al., 2005) and referred to in the discussion.

conformation of His311 (see below) does not change. In contrast, we detected no new conformations of loops 1-3 in molecule $B$ relative to $1 \operatorname{logs}$ and $1 \mathrm{y} 7 \mathrm{v}$, but did detect a novel combination of previously observed conformations of the loops.

3.3.2. Active site. Table 5 shows that the distances between Glu340 and Glu235, the catalytic site residues of GCase, are $\sim 5 \AA$, which is consistent with a retaining mechanism (Davies \& Henrissat, 1995) of catalytic activity. It is also seen from this table that the average distances between $\mathrm{O}^{\varepsilon 1} \ldots \mathrm{O}^{\varepsilon 1}$ and $\mathrm{O}^{\varepsilon 2} \cdots \mathrm{O}^{\varepsilon 2}$ are similar in all the crystal structures reported to date. The differences in distances between individual carboxylate groups of the catalytic residues of $2 \mathrm{j} 25$ and of 1 ogs and $1 \mathrm{y} 7 \mathrm{v}$ arise from changes in $\chi_{2}$ torsion angles. Since the acidic $\mathrm{p} K_{\mathrm{a}}$ of the $\mathrm{pH}$-activity profile is around $\mathrm{pH} 4.7$ (Liou et $a l ., 2006)$ and the $\mathrm{pH}$ values for the crystal structures examined here lie on either side of this $\mathrm{p} K_{\mathrm{a}}$ value (Dvir et al., 2003; Premkumar et al., 2005), it is plausible that the observed differences are related to the protonation states of the activesite carboxyl groups. Thus, 1 ogs and $1 \mathrm{y} 7 \mathrm{v}$, which were both

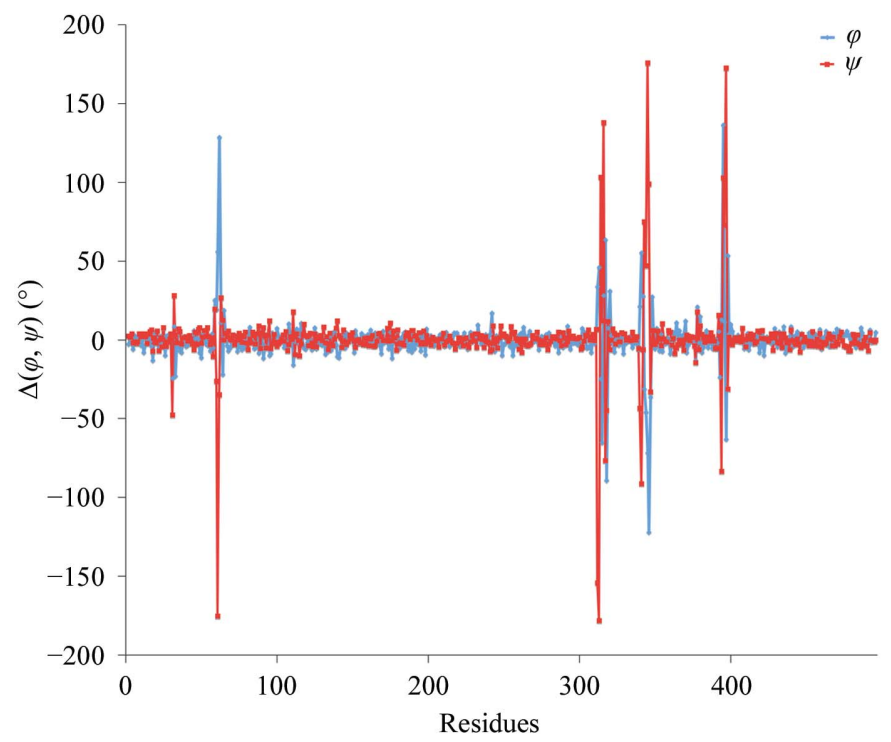

Figure 3

A $\varphi, \psi$ difference plot between main-chain angles in molecules of the asymmetric unit of GCase (PDB code 2j25). The differences between angles are constrained to be between $-180^{\circ}$ and $+180^{\circ}$.
Table 5

Interatomic distances between side chains of catalytic residues.

Distances are shown in $\AA$ for the following residues: Glu235-Glu340 $\left(\mathrm{O}^{\varepsilon 1} \cdots \mathrm{O}^{\varepsilon 1}\right)$, Glu235-Glu340 $\left(\mathrm{O}^{\varepsilon 2} \cdots \mathrm{O}^{\varepsilon 2}\right)$ and Glu235-His311 $\left(\mathrm{O}^{\varepsilon 2} \cdots \mathrm{N}^{\delta 1}\right)$. The average distance is between the carboxyl $\mathrm{O}$ atoms of the catalytic glutamates. In the case of 1 nof, the distances are measured between residue Glu165 and residues Glu253 and His230.

\begin{tabular}{llllll}
\hline & $\mathrm{O}^{\varepsilon 1} \cdots \mathrm{O}^{\varepsilon 1}$ & $\mathrm{O}^{\varepsilon 2} \cdots \mathrm{O}^{\varepsilon 2}$ & $\begin{array}{l}\text { Average } \\
\text { distance }\end{array}$ & $\begin{array}{l}\text { Closest } \\
\text { approach }\end{array}$ & $\mathrm{O}^{\varepsilon 2} \cdots \mathrm{N}^{\delta 1}$ \\
\hline 1ogs- $A$ & 5.9 & 4.2 & 5.05 & 4.2 & 2.7 \\
1 ogs- $B$ & 6.2 & 4.3 & 5.25 & 4.3 & 2.8 \\
$1 \mathrm{y} 7 \mathrm{v}-A$ & 6.2 & 4.0 & 5.10 & 4.0 & 2.8 \\
$1 \mathrm{y} 7 \mathrm{v}-B$ & 6.1 & 3.9 & 5.00 & 3.9 & 2.9 \\
$2 \mathrm{j} 25-A$ & 4.6 & 4.2 & 4.40 & 4.2 & 3.2 \\
$2 \mathrm{j} 25-B$ & 4.9 & 4.1 & 4.50 & 4.1 & 3.1 \\
$2 \mathrm{f} 61-A$ & 5.0 & 5.2 & 5.10 & 4.7 & 3.4 \\
$2 \mathrm{f} 61-B$ & 6.3 & 3.9 & 5.10 & 3.9 & 2.6 \\
1 nof & $5.8 \dagger$ & $4.2 \dagger$ & 5.00 & 4.2 & 2.6 \\
\hline
\end{tabular}

$\dagger$ The numbering of $\mathrm{O}^{\varepsilon 1}$ and $\mathrm{O}^{\varepsilon 2}$ is arbitrary and the assignment in 1nof was therefore altered so as to correspond to the numbering in the other structures.

crystallized at $\mathrm{pH} 4.6$, would be expected to have both carboxyl groups protonated. This would explain both the greater inter-residue distances and changes in $\chi_{2}$ torsion angles relative to $2 \mathrm{j} 25$. The latter, having been crystallized at $\mathrm{pH} 5.5$, would be expected to have only one carboxyl group protonated, which should allow closer approach of the two carboxylates.

3.3.3. Anion-binding sites. In GCase, there are two clusters of $\mathrm{SO}_{4}^{2-}$ ions, which were previously detected in $10 \mathrm{gs}$ and $1 \mathrm{y} 7 \mathrm{v}$ but not analyzed in detail (Fig. $6 a$ ); only one of these clusters was described in $2 \mathrm{f} 61$ (Liou et al., 2006). Each cluster contains 2-3 $\mathrm{SO}_{4}^{2-}$ ions. One cluster is located close to residues 12,44 , 45, 353 and 356-358, near the active site (Fig. 6a). The second is near residues 79, 228, 277 and 306. Since the negative charge

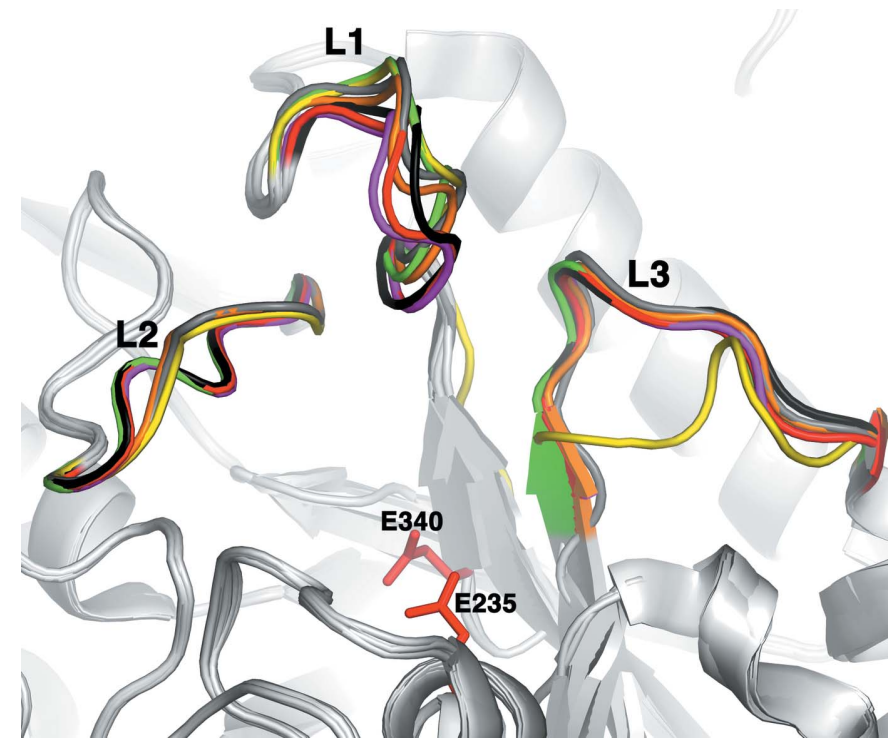

Figure 4

Conformational classification of loops 1, 2 and 3 (Kleywegt, 1996). The structures of the loops of 2j25- $A$ (GCase) are in yellow, 2j25- $B$ in green, $1 \mathrm{y} 7 \mathrm{v}$ (pDG-GCase) in magenta, 1ogs- $A$ (pDG-GCase) in orange, 1ogs- $B$ in red, 2f61- $A$ (pDG-GCase) in black and $2 \mathrm{f} 61-B$ in grey. Catalytic residues are in red. 
of $\mathrm{SO}_{4}^{2-}$ is similar to that of the negatively charged phospholipids required for optimal GCase activity in vivo (Grace et al., 1994), we suggest that the $\mathrm{SO}_{4}^{2-}$ cluster adjacent to the active site may be involved in the membrane association of GlcCerase (Fig. 6b).

\subsection{Comparison of the active sites of GCase and xylanase}

We compared the structure of GCase with that of xylanase (PDB code 1nof; Larson et al., 2003), a member of glycoside

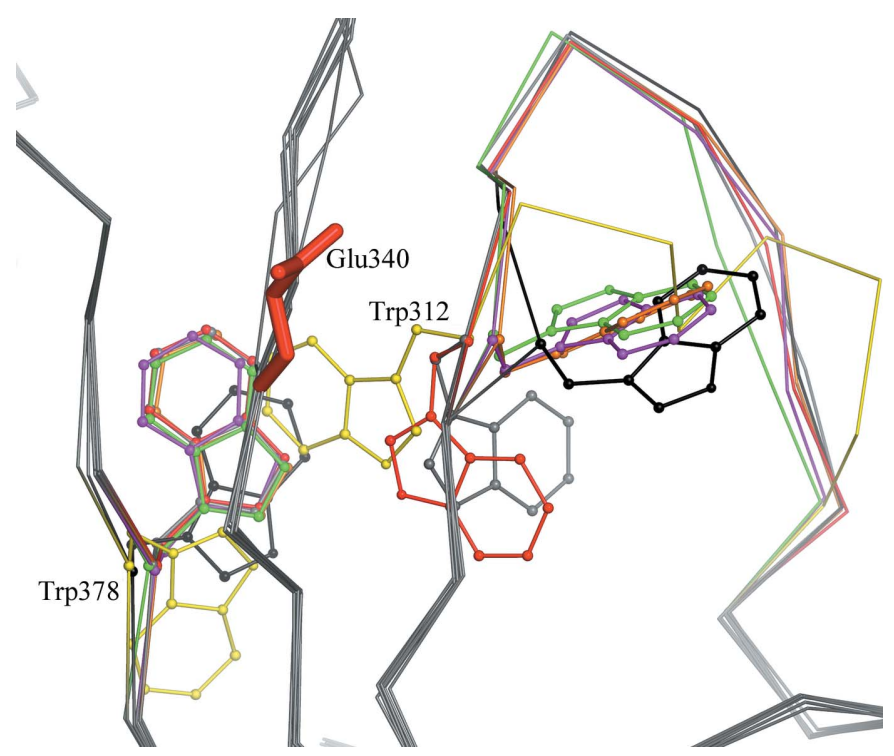

Figure 5

Conformational changes in $\operatorname{Trp} 312$ and $\operatorname{Trp} 378$ and movements in the backbone near the active site. Yellow, $2 \mathrm{j} 25-A$; green, $2 \mathrm{j} 25-B$; orange, 1ogs$A$; red, 1ogs- $B$; black, 2f61- $A$; grey, 2f61- $B$; magenta, $1 \mathrm{y} 7 \mathrm{v}$; catalytic residue in red. hydrolase family 5 , which shows the highest sequence identity to GlcCerase of all structures in the PDB. In order to crossvalidate the positioning and distances of the catalytic residues, the GCase structure was aligned with that of xylanase (Fig. 7). Comparison of the active sites was attained by aligning the catalytic residues: Glu235 and Glu340 in GCase and Glu165 and Glu253 in xylanase. This alignment gave nine identical and two similar residues within $5 \AA$ of the catalytic residues, which align virtually identically. Only one residue, Tyr313 in GCase, which corresponds to Tyr232 in xylanase, shows conformational variability.

Based on the structural and sequence alignment, His311 in GCase corresponds to His230 in xylanase. The distances between His311 and Glu235 in GCase and between His230 and Glu165 in xylanase are compatible with hydrogen bonding. This conserved histidine residue may introduce additional hydrogen-bond coordination between the catalytic residues and thus play a role in stabilizing the active sites of both GCase and xylanase.

\section{Discussion}

The major conclusion of the current study is that partial deglycosylation of Cerezyme ${ }^{\circledR}$ (pDG-GCase) by $\mathrm{N}$-glycosidase $\mathrm{F}$ has no major effect on the conformation of GlcCerase, thus validating our previous structural analyses of crystals obtained after $\mathrm{N}$-glycosidase $\mathrm{F}$ treatment.

We observe a significant number of Asn-linked glycans in the GCase structure. The packing of the protein molecules in the crystal allows the insertion of sugar chains into the free cavities, which are sufficiently large to accommodate the glycans without introducing steric clashes with symmetryrelated molecules. We are able to observe two large segments of two glycan chains, those attached to Asn19-B and Asn59-B,

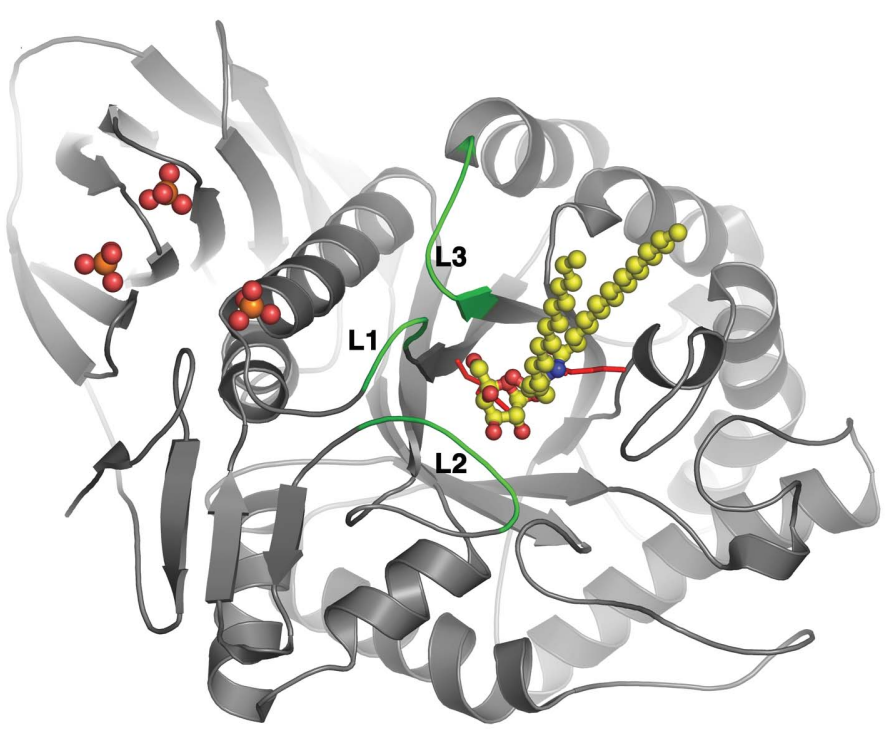

(a)

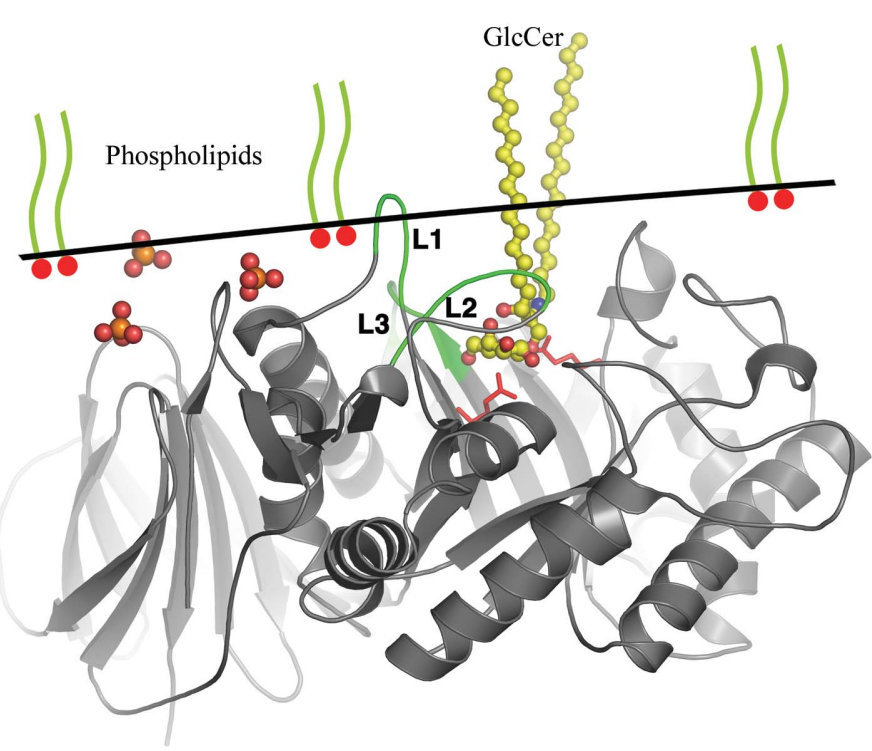

(b)

Figure 6

Putative membrane binding sites in crystal structures of GlcCerase. Catalytic residues are shown in red and loops 1-3 in green. Substrate binding was modelled according to Dvir et al. (2003) and shown in yellow. (a) Some of the sulfates are shown in space-filling representation in red. (b) Side-on view [rotated $\sim 90^{\circ}$ around the horizontal axis relative to $(a)$ ] of a putative mode of binding of GlcCerase to negatively charged phospholipids. 
Table 6

Comparison of GlcCerase monomers in various crystal structures.

R.m.s. deviations are shown in $\AA$.

\begin{tabular}{lllllll}
\hline & 1 logs- $A$ & 1 logs- $B$ & $1 \mathrm{y} 7 \mathrm{v}-A$ & $1 \mathrm{y} 7 \mathrm{v}-B$ & $2 \mathrm{f} 61-A$ & 2f61- $B$ \\
\hline $2 \mathrm{j} 25-A$ & 0.35 & 0.37 & 0.34 & 0.34 & 0.37 & 0.39 \\
$2 \mathrm{j} 25-B$ & 0.37 & 0.34 & 0.33 & 0.31 & 0.39 & 0.35 \\
$2 \mathrm{f} 61-A$ & 0.19 & 0.33 & 0.30 & 0.33 & & \\
$2 \mathrm{f} 61-B$ & 0.31 & 0.17 & 0.30 & 0.28 & & \\
$1 \mathrm{y} 7 \mathrm{v}-A$ & 0.25 & 0.26 & & & & \\
$1 \mathrm{y} 7 \mathrm{v}-B$ & 0.27 & 0.24 & & & & \\
\hline
\end{tabular}

which are in contact with each other and thus mutually stabilize each other's conformations. It was previously reported that the visualization of a sequence of seven sugars of a glycan chain in Erythrina corallodendron lectin could similarly be ascribed to its immobilization by a symmetry-related molecule, whereas only chitobioses could be detected on chains that were not stabilized by such intermolecular interactions (Shaanan et al., 1991).

The observation of sugar residues attached to Asn19 in pDG-GCase suggested that this residue may be inaccessible to enzymatic cleavage by $N$-glycosidase $\mathrm{F}$. This was verified by molecular modelling, which was performed to determine whether all four N-linked sites are available for glycosylation in the monomer and whether the glycans modelled at these sites block access to the active site. Modelling of the crystal packing for pDG-GCase showed that except for Asn19, the putative glycosylation sites could not be occupied with the observed Asn side-chain conformations. According to the modelling, a glycan attached to Asn146- $A$ in pDG-GCase with

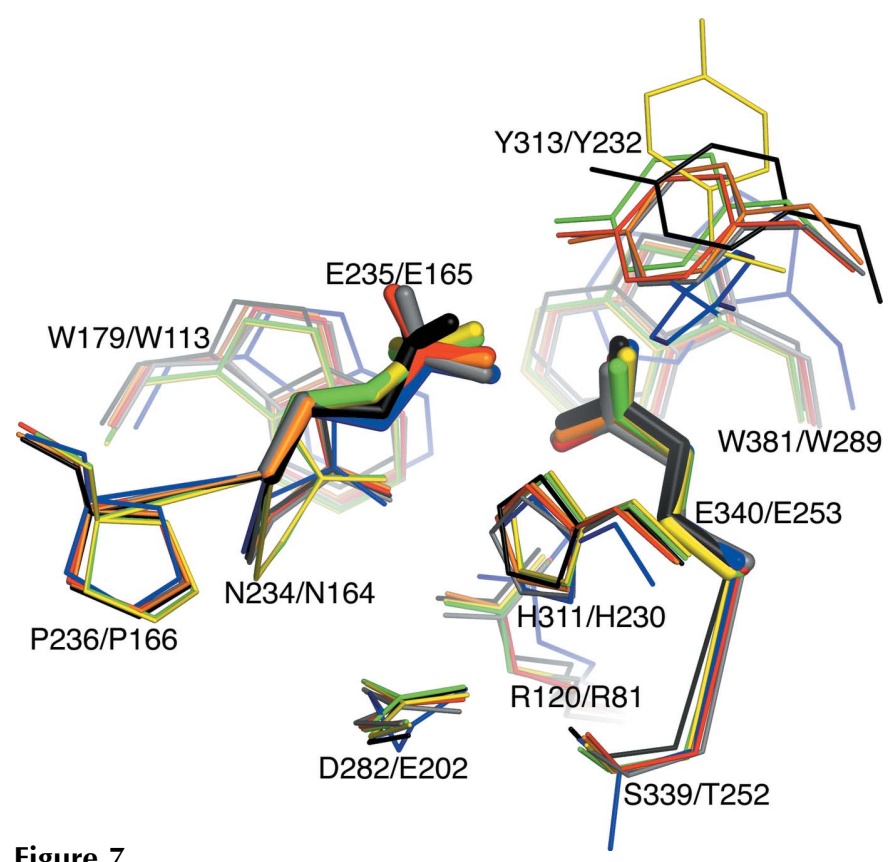

Figure 7

Comparison of the active sites of GCase and xylanase. Residues within a $5 \AA$ radius of the active site are displayed. Yellow, $2 \mathrm{j} 25-A$ (GCase); green, $2 \mathrm{j} 25-B$; blue, 1nof (xylanase); orange, 1ogs- $A$ (pDG-GCase); red, 1ogs- $B$; black, 2f61- $A$ (pDG-GCase); grey, 2f61- $B$. The first number refers to residue in GCase and the second number to the corresponding residue in xylanase. the observed side-chain torsion angles (Petrescu et al., 2004) would clash sterically with a glycan attached to Asn146-B, indicating that both sites cannot be occupied simultaneously. Similarly, a core glycan chain modelled on Asn19- $B$ would clash with a modelled glycan on Asn59 of the adjacent $B$ chain. Thus, the fact that well resolved electron density was seen for a glycan on Asn19-B in pDG-GCase implies that Asn59 must be unoccupied. The presence of glycans on Asn59 and Asn146 in the new GCase structure causes changes in the $\chi_{1}$ and $\chi_{2}$ torsion angles of the Asn side chains (Petrescu et al., 2004), thus eliminating steric clashes.

Despite the differences in crystallization conditions and glycosylation patterns, there are only minor differences between the pDG-GCase and GCase structures, which have a similar asymmetric unit and crystal packing, as confirmed by the low r.m.s. deviations (Table 6). However, several small but important differences were detected in the conformation of the lid in molecule $A$ of GCase, encompassed by loops 1-3 (Premkumar et al., 2005). Importantly, these differences cannot be ascribed to glycosylation, since molecule $B$ in the GCase structure shows conformations of loops 1-3 that are identical to those seen in pDG-GCase. Molecule $A$ in GCase displays some additional changes near the active site relative to pDG-GCase, in residues Trp312, Trp378 and 341-344. Most significant are the changes in Trp312 and Trp378, whose sidechain conformations depend on the conformation of loop 3; as a result, the conformations of residues 341-344 are also altered. It should be stressed that even though these loops control access to the active site, substrate binding must produce a conformational change in the loops in order to allow the process to occur without the introduction of steric clashes, similar to that seen in glycosyl transferases (Qasba et al., 2005).

Comparison of the catalytic residues Glu235 and Glu340 in the various GlcCerase structures reveals minor positional differences, some of which arise from changes in the torsion angles of Glu235. The side-chain conformation of Glu235 depends on interactions with surrounding residues via hydrogen bonds. This may explain the differences in the distances between Glu235 and Glu340 in the various structures solved to date (Table 5). Moreover, it was recently implied that the structure of pDG-GCase may not be valid owing to the loss of activity caused by $N$-glycosidase F treatment (Liou et al., 2006). That this is not the case is supported by the similarity in distances between Glu235 and Glu340 ( $1 \mathrm{ogs}, 1 \mathrm{y} 7 \mathrm{v}$ and $2 \mathrm{j} 25$ versus $2 \mathrm{f} 61$ ). Moreover, we have shown that pDG-GCase is fully catalytically active (Premkumar et al., 2005). Unfortunately, no data are presented to document the purported loss of activity produced by $\mathrm{N}$-glycosidase F treatment (Liou et al., 2006). In addition, considerable concern remains about the lack of correlation between the electron density and the reported coordinates in some regions of $2 \mathrm{f} 61$ (Liou et al., 2006). Irrespective of these concerns, the structures of $2 \mathrm{f} 61$ and of $1 \mathrm{ogs}, 1 \mathrm{y} 7 \mathrm{v}$ and $2 \mathrm{j} 25$ are all very similar, with the minor exceptions noted above with respect to $2 \mathrm{j} 25$.

In summary, both the pDG-GCase and GCase structures reported by us are fully compatible with the catalytic 
mechanism of GlcCerase and provide the molecular tools for detailed structure-function analysis, which may lead to improved GlcCerase for use in enzyme-replacement therapy.

This work was supported by the Horowitz Foundation, the National Gaucher Foundation, the Oxford Glycobiology Institute, the Yeda fund of the Weizmann Institute, the Divadol Foundation, the Bruce Rosen Foundation, the Kalman and Ida Wolens Foundation, the Jean and Jula Goldwurm Memorial Foundation, the Kimmelman Center for Biomolecular Structure and Assembly, the Benoziyo Center for Neuroscience and the Minerva Foundation. We thank the staff at beamline ID23eh1 at the ESRF synchrotron in Grenoble for assistance during data collection, Dr Orly Dym for help in examining the structures and Dr Harry Greenblatt and Yehudit Hasin for commenting on the manuscript. JLS is the Morton and Gladys Pickman Professor of Structural Biology and AHF is the Joseph Meyerhoff Professor of Biochemistry.

\section{References}

Altschul, S. F., Madden, T. L., Schaffer, A. A., Zhang, J., Zhang, Z., Miller, W. \& Lipman, D. J. (1997). Nucleic Acids Res. 25, 3389-3402.

Berman, H. M., Westbrook, J., Feng, Z., Gilliland, G., Bhat, T. N., Weissig, H., Shindyalov, I. N. \& Bourne, P. E. (2000). Nucleic Acids Res. 28, 235-242.

Beutler, E. \& Grabowski, G. A. (2001). The Metabolic and Molecular Bases of Inherited Disease, edited by C. R. Scriver, W. S. Sly, B. Childs, A. L. Beaudet, D. Valle, K. W. Kinzler \& B. Vogelstein, pp. 3635-3668. New York: McGraw-Hill.

Brady, R. O. (2006). Annu. Rev. Med. 57, 283-296.

Bruhn, H. (2005). Biochem. J. 389, 249-257.

Chayen, N. E. (1998). Acta Cryst. D54, 8-15.

Collaborative Computational Project, Number 4 (1994). Acta Cryst. D50, 760-763.

D'Arcy, A., Mac Sweeney, A., Stihle, M. \& Haber, A. (2003). Acta Cryst. D59, 396-399.

Davies, G. \& Henrissat, B. (1995). Structure, 3, 853-859.

Dvir, H., Harel, M., McCarthy, A. A., Toker, L., Silman, I., Futerman, A. H. \& Sussman, J. L. (2003). EMBO Rep. 4, 704-709.
Emsley, P. \& Cowtan, K. (2004). Acta Cryst. D60, 2126-2132.

Futerman, A. H. \& van Meer, G. (2004). Nature Rev. Mol. Cell Biol. 5, 554-565.

Grabowski, G. A., Gatt, S. \& Horowitz, M. (1990). Crit. Rev. Biochem. Mol. Biol. 25, 385-414.

Grace, M. E., Newman, K. M., Scheinker, V., Berg-Fussman, A. \& Grabowski, G. A. (1994). J. Biol. Chem. 269, 2283-2291.

Han, K. K. \& Martinage, A. (1992). Int. J. Biochem. 24, 19-28.

Horowitz, M., Wilder, S., Horowitz, Z., Reiner, O., Gelbart, T. \& Beutler, E. (1989). Genomics, 4, 87-96.

Jmoudiak, M. \& Futerman, A. H. (2005). Br. J. Haematol. 129, 178-188.

Kabsch, W. (1993). J. Appl. Cryst. 26, 795-800.

Keen, N. T., Boyd, C. \& Henrissat, B. (1996). Mol. Plant Microbe Interact. 9, 651-657.

Kleywegt, G. J. (1996). Acta Cryst. D52, 842-857.

Larson, S. B., Day, J., Barba de la Rosa, A. P., Keen, N. T. \& McPherson, A. (2003). Biochemistry, 42, 8411-8422.

Laskowski, R. A., Moss, D. S. \& Thornton, J. M. (1993). J. Mol. Biol. 231, 1049-1067.

Liou, B., Kazimierczuk, A., Zhang, M., Scott, C. R., Hegde, R. S. \& Grabowski, G. A. (2006). J. Biol. Chem. 281, 4242-4253.

Lutteke, T., Frank, M. \& von der Lieth, C. W. (2005). Nucleic Acids Res. 33, D242-D246.

Lutteke, T. \& von der Lieth, C. W. (2004). BMC Bioinformatics, 5, 69.

McCoy, A. J., Grosse-Kunstleve, R. W., Storoni, L. C. \& Read, R. J. (2005). Acta Cryst. D61, 458-464.

Murray, G. J. (1987). Methods Enzymol. 149, 25-42.

Murshudov, G. N., Vagin, A. A. \& Dodson, E. J. (1997). Acta Cryst. D53, 240-255.

Petrescu, A. J., Milac, A. L., Petrescu, S. M., Dwek, R. A. \& Wormald, M. R. (2004). Glycobiology, 14, 103-114.

Premkumar, L., Sawkar, A. R., Boldin-Adamsky, S., Toker, L., Silman, I., Kelly, J. W., Futerman, A. H. \& Sussman, J. L. (2005). J. Biol. Chem. 280, 23815-23819.

Qasba, P. K., Ramakrishnan, B. \& Boeggeman, E. (2005). Trends Biochem. Sci. 30, 53-62.

Sawkar, A. R., Adamski-Werner, S. L., Cheng, W. C., Wong, C. H., Beutler, E., Zimmer, K. P. \& Kelly, J. W. (2005). Chem. Biol. 12, $1235-1244$.

Shaanan, B., Lis, H. \& Sharon, N. (1991). Science, 254, 862-866.

Vaccaro, A. M., Tatti, M., Ciaffoni, F., Salvioli, R., Barca, A. \& Scerch, C. (1997). J. Biol. Chem. 272, 16862-16867.

Wormald, M. R., Petrescu, A. J., Pao, Y. L., Glithero, A., Elliott, T. \& Dwek, R. A. (2002). Chem. Rev. 102, 371-386. 